\title{
A pilot study: a possible implication of Candida as an etiologically endogenous pathogen for oral lichen planus
}

\author{
Hong $\mathrm{He}^{1,2^{* \dagger}} \mathbb{D}$, Xinyu Xia ${ }^{1}$, Haiping Yang ${ }^{1,2 \dagger}$, Qiao Peng ${ }^{3 \dagger}$ and Jiaoer Zheng ${ }^{1,2^{*}}$
}

\begin{abstract}
Background: The aim of this study was to investigate the prevalence and genotypic profiles of Candida albicans in patients with oral lichen planus (OLP).

Materials and methods: Positive rates and genotypic profiles of Candida albicans strains from OLP patients and healthy controls were analyzed. Random amplified polymorphic DNA and internal transcribed spacer of ribosome DNA polymerase chain reactions were used to sequence the DNA of these strains, and then their genetic similarity was measured using BLAST, UIV Band, and Vector NTI Suite Sequence Analyses Software.

Results: The prevalence of $C$. albicans strains detected from erosive-OLP, non-erosive OLP, and normal individuals was 18.87, 18.75 , and $7.92 \%$, respectively. Four different genotypes were revealed by the two methods. To be specific, type I was found only in the healthy subjects; type II a and II b were found in non-erosive OLP, and type III was identified in erosive OLP. Intragroup similarity coefficients, i.e. $S_{A B}$ were $100 \%$, and inter-groups similarity coefficients, i.e. $S_{A B}$ were less than $30 \%$.

Conclusions: The genotypic results of $C$. albicans in OLP revealed an endogenous rather than exogenous infection of $C$. albicans. In addition, a possible pathogenic role of C. albicans in OLP, with the etiologic sense contributing to a more proper recognition on the pathogenesis, development, and progression of OLP, as well as some strategies for its diagnosis and treatment were identified.
\end{abstract}

Keywords: Oral lichen planus, Etiology, Endogenous infection, Candida albicans, ITS sequence, Homology similarity coefficient $S_{A B}$

\section{Background}

Oral lichen planus(OLP)is a common chronic inflammatory oral mucosal disease affecting $1-2 \%$ of the general population [1-3]. It is considered as a potentially malignant disorder with a deteriorated transformation prevalence of $0-1 \%$ according to the World Health Organization $[4,5]$. While the etiology of OLP remains to be elucidated, the existing evidence suggests that microbial infection, psychological disorders, allergies, and immunodeficiency are closely associated with the pathogenesis of OLP [5-7].

Considering the oral microorganisms, Candida species are the commensal fungus in the mucosal flora of healthy

\footnotetext{
* Correspondence: honghehh@zju.edu.cn; rainbow050607@126.com Hong He, Haiping Yang and Qiao Peng are first co authors.

${ }^{1}$ The Affiliated Stomatology Hospital, Zhejiang University School of Medicine, Yan'an Road, Hangzhou 310006, China

Full list of author information is available at the end of the article
}

individuals, which also have a crucial role in OLP [8-10]. Among Candida species, Candida albicans (C. albicans) is the predominant specie in the OLP patients [11-13]. Arora et al. have investigated the prevalence and phenotypic variation of Candida species in OLP cases, and found that $C$. albicans constituted the majority of the five identified Candida species [12]. Thus, C. albicans from OLP has become a popular research topic over recent years in many clinical and laboratory studies.

Initially, the researches were focused on the phenotypic presence of $C$. albicans in OLP; however, due to the low resolution of phenotypic analysis they failed to answer the following basic questions: whether the pathogen is a fungus; whether $C$. albicans is an initial pathogen or a symbiont; whether it facilitate the infection in OLP, or vice versa; and whether $C$. albicans is a probable antigen for OLP. It is worth noting that a close correlation might exist

(c) The Author(s). 2020 Open Access This article is distributed under the terms of the Creative Commons Attribution 4.0 International License (http://creativecommons.org/licenses/by/4.0/), which permits unrestricted use, distribution, and 
between genotypic profiles and virulence attributes [14]. Moreover, genotyping can provide information about DNA and has stronger resolution than phenotyping necessary to distinguish variants among individuals or stages $[14,15]$. Therefore, the relationship between genotypes of C. albicans and OLP needs to be further investigated.

In this study, genotyping analyses using BLAST, UIV Band, and Vector NTI Suite Sequence Analysis software were conducted to investigate the relationship between OLP and genotypes of C. albicans. Combination of random amplified polymorphic DNA (RAPD) and internal transcribed spacer (ITS) $[10,16,17]$ of ribosome DNA polymerase chain reaction (PCR) was used to intergroup genotype $C$. albicans isolates from healthy individuals and OLP patients. The results showed that the phenotypes and the intragroup genotype of C. albicans were homologous; while they were genotypically heterogeneous among erosive (E)-OLP, non-erosive (NE)-OLP and healthy individuals. Both methods revealed three discrepant subtypes of $C$. albicans in clinical different stages of OLP, and one different subtype in healthy controls. Thus, we assumed that endogenous infection rather than exogenous infection of $C$. albicans might be the vital factor in OLP etiology.

\section{Materials and methods}

This study was approved by the local research ethics committees, the ethics committee of the Affiliated Second Hospital, and the ethics committee of the Affiliated Stomatology Hospital, Zhejiang University School of Medicine, China. All patients provided written informed consent. The protocols were reviewed and approved by the local institutional review boards (IRB). The IRB numbers for our medical ethic files were No. 2015 (17) of the stomatology hospital and 2010 (137) of the second hospital both affiliated to School of Medicine Zhejiang University. All participants underwent training session to minimize the undesirable discrepancy.

G*power software was used for power calculation; a power was $94 \%$ with $\alpha=0.05$ for the standard deviation in groups of patients and control. A total of 250 subjects as sample size (aged between 16 and 86 years), including 149 patients with OLP and 101 agesex-matched healthy volunteers $(P>0.05)$, were recruited at the Affiliated Hospitals of Zhejiang University between February 2010 and March 2016 (Table 1 and Fig. 1). The patients were diagnosed with OLP based on clinical and histopathological criteria, as well as the criteria of Chinese Stomatological Association of Oral Medicine. The 101 healthy individuals had no oral mucosa diseases. The exclusion criteria were as follows: patients having full or partial dentures, those taking broad-spectrum antibiotics, antifungals, glucocorticoids, or immunosuppressive agents over a long period of time or within last 3 months, and those
Table 1 Clinical features and positive culture rate of Candida albicans strains of the subjects $\left({ }^{* *} P=0.024,{ }^{*} P=0.044\right.$ )

\begin{tabular}{|c|c|c|c|c|c|}
\hline \multirow[t]{2}{*}{ Groups } & \multicolumn{2}{|c|}{ Gender } & \multirow{2}{*}{$\begin{array}{l}\text { Age } \\
\text { (Mean) }\end{array}$} & \multirow{2}{*}{$\begin{array}{l}\text { Positive } \\
\text { cases }\end{array}$} & \multirow{2}{*}{$\begin{array}{l}\text { Positive } \\
\text { rate }\end{array}$} \\
\hline & M & $\mathrm{F}$ & & & \\
\hline $\mathrm{NE}-\mathrm{OLP}(n=96)$ & 47 & 49 & $48.22 \pm 16.43$ & 18 & $18.75 \%{ }^{* *}$ \\
\hline $\mathrm{E}-\mathrm{OLP}(n=53)$ & 27 & 26 & $51.37 \pm 15.78$ & 10 & $18.87 \%{ }^{*}$ \\
\hline Controls $(n=101)$ & 51 & 50 & $49.69 \pm 17.19$ & 8 & $7.92 \%$ \\
\hline
\end{tabular}

with systemic diseases, including diabetes, thyroid hypofunction, and immune deficiency.

\section{Isolates}

From experience, the authors have thought that swabbing is better than rinse to isolate Candida species from the oral cavity. All the sample swabs were taken from subjects' oral mucosal membrane. Lesion regions in patients with OLP and eight regions in the healthy individuals' oral mucosa were consistently swabbed. The eight regions in healthy individuals' oral mucosa were bilateral buccal, palate, back and two sides of abdominal tongue, and vestibules. The team members were trained for standard performance actions. The clinical isolates were randomly labeled, and researchers who conducted phenotyping and genotyping analyses were blinded to all information of the isolates. All isolates were first analyzed using conventional microbiological identification methods [18, 19], and those with the morphological characteristics of C. albicans were analyzed using germ tube test and the API 20C AUX identification kit (Analytical Profile Index; BioMe'rieux S.A., France).

\section{DNA extraction}

DNA was extracted using the Biospin Fungus Genomic DNA Extraction Kit (Bioflux, China) following the manufacturer's protocol. DNA concentration was examined using the Ultraviolet Spectrophotometer (Pharmacia Biotech, USA, 07450).

\section{RAPD fingerprinting and $S_{A B}$ analysis}

RAPD was conducted as described [20]. Two primers (C1: 5'-ACGGTACACT-3'; C2: 5'-GTTCCGCCC-3') were used in this study. After amplification, the products were electrophoresed in $2 \%$ of agarose gel. After staining with ethidium bromide (Kaiser Germany), the PCR products were qualified using the UIV Band system. Information about the location and relative molecular mass of the main amplified bands was extracted for calculating the genetic similarity coefficient $\mathrm{S}_{\mathrm{AB}}$ between any two bands. Strains were categorized into several families that share gene homology at a criterion of $\mathrm{S}_{\mathrm{AB}}=0.8$. 


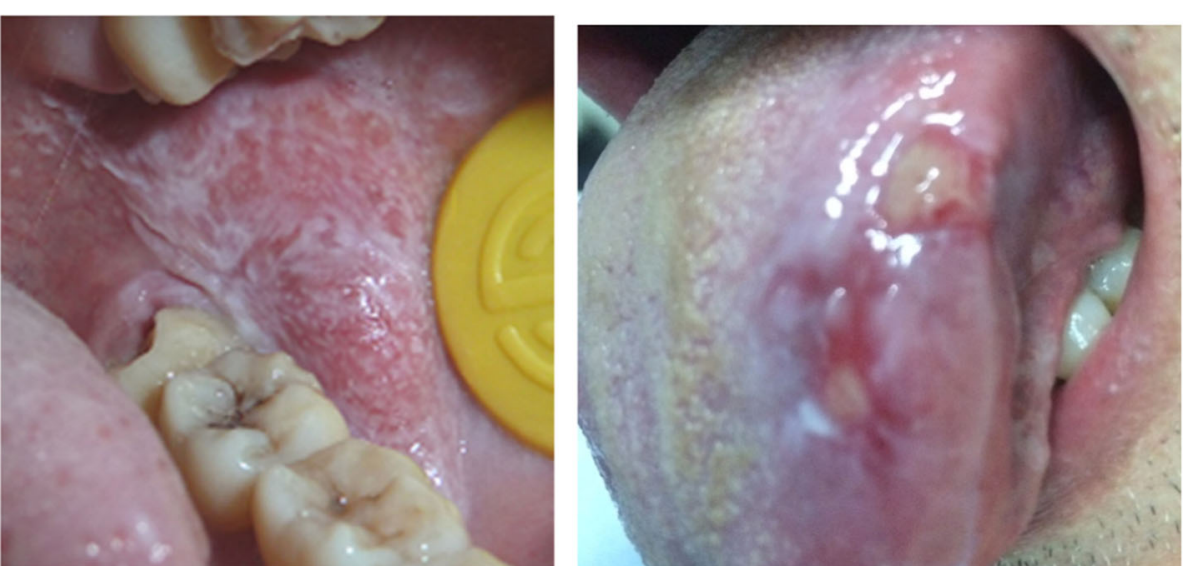

Fig. 1 OLP Lesions in left buccal (a non-erosive one) and left tongue (an erosive one)

\section{ITS sequence determination}

ITS analysis was conducted as described in a previous study (White et al.). PCR amplification was performed with primers (5'-GGAAGTAAAAGTCGTAACAAGG-3'; 5' GCTGCGTTCTTCATCGATGC-3') that were designed based on the conserved regions of ITS 1 and ITS 2 rRNA genes. The amplified DNA fragment included the intervening 5.8S gene and the ITS 1 and ITS 2 noncoding regions. Primers were synthesized by the Eppley Molecular Biology Core Laboratory (NE, USA). The amplified PCR products were purified and sequenced by TaKaRa Bio-Engineering Co., Ltd. (Dalian, China). BLAST was used to compare the DNA sequence with standard ITS sequences of $C$. albicans in the GenBank at National Center for Biotechnology Information to determine the taxonomy of the isolates. Intraspecies sequence similarity and variation for isolates were determined using the Vector NTI Suite software (www. liax.cn) and visually confirmed using pairwise nucleotide alignments. Referenced isolates were also aligned. The similarities of the sequences were determined with the expectation frequency minimized to 0.0001 .

\section{Statistical analysis}

A $P$ value $<0.05$ was set as the standard statistical significance. All calculations were performed by $X^{2}$ test using SPSS statistical software (SPSS 25.0; SPSS Inc., Chicago, IL, USA).

\section{Results}

Positive rate of $C$. albicans culture-prevalence of $C$. albicans in OLP

The results showed that $C$. albicans were identified from 16 of 96 NE-OLP (16.67\%), 10 of 53 E-OLP (18.87\%), and 8 of 101 healthy controls (7.92\%) (Table 1). The prevalence of $C$. albicans in NE-OLP and E-OLP were significantly higher than that in the healthy symbiotic group ( $X^{2}$ test, $P<0.05$ respectively as 0.024 and 0.044 ), but no significant difference was found between NE-OLP and E-OLP ( $X^{2}$ test, $P>0.05$ ) (Table 1$)$. Using germ tube test and the API 20C AUX identification kit, eight isolates of C. albicans from NE-OLP, two from E-OLP, and two from the healthy controls were identified.

\section{RAPD and UIV band analysis}

Electrophoresis analysis of RAPD products showed that the healthy controls had only one band, both E-OLP isolates had multiple bands, and all the eight NE-OLP isolates had two bands (Fig. 2). $\mathrm{S}_{\mathrm{AB}}$ analysis further showed that the NE-OLP formed a major clade, and the E-OLP formed another separate clade. The health control was basal to the NE-OLP and E-OLP clades.

Then, these RAPD genomes were analyzed by UIV Band. The results showed that genetic homology coefficient $\mathrm{S}_{\mathrm{AB}}$ was less than $30 \%$ between the control, NE-OLP, and E-OLP groups, while it was $100 \%$ in a single subgroup. Additionally, the NE-group could be categorized into two subgroups, namely two genotypes, one including strains numbered $3-7$ and 11 , and the other including strains numbered 9, 10 and 12 (Fig. 3).

\section{ITS sequences}

The DNA fragment with expected size (250 bp) was successfully amplified from all isolates (Fig. 4). BLAST analyses of the sequences referring to NCBI databases clearly showed that all isolates belonged to the C. albicans species. Then, these ITS sequences were genotyped using Vector NTI Suite software, and the sequence analysis showed that the base pair genotype results of ITS regions of the strains were consistent with the results of the RAPD analysis (Fig. 5). Specifically, the type I was found from normal strains, type IIa and IIb were 

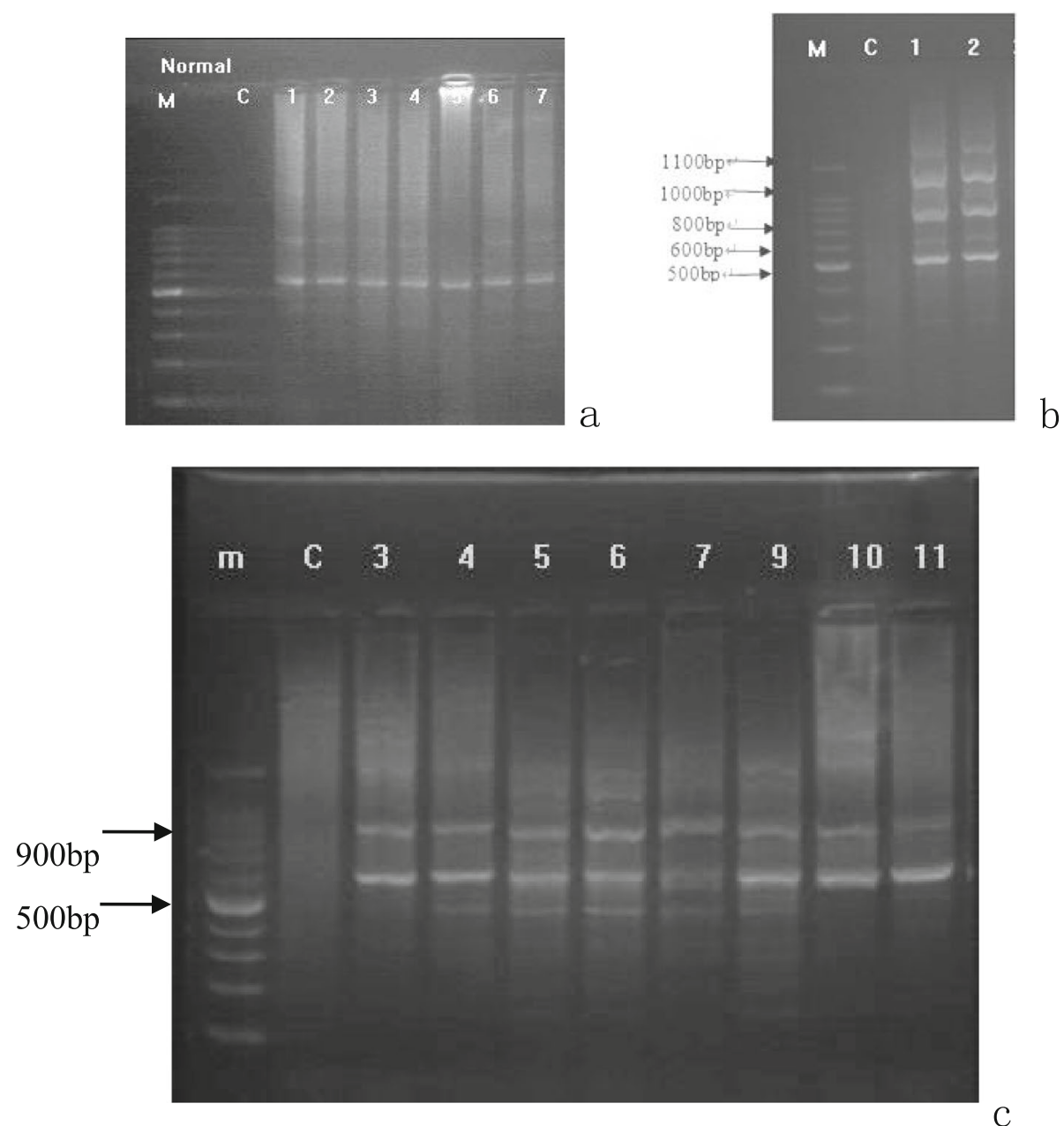

Fig. 2 Electrophoresis pattern of RAPD results of C. albicans from the healthy group (2a: 1-7 electrophoresis lanes), E-OLP group (2b: 1-2 electrophoresis lanes), and NE-OLP group (2c: 3-11 electrophoresis lanes). M, Marker; C, control. (The numbers of strains did not relate to the numbers of cases)

detected from NE-OLP cases, and type III was identified from E-OLP cases.

\section{Discussion}

The relationship between C. albicans and the etiology of OLP has long been puzzling many researchers and it still remains to be elucidated. The present study revealed that $C$. albicans from OLP were quantitatively and qualitatively distinctive with heathy individuals. Moreover, $C$. albicans isolates from both NE-OLP and E-OLP were genetically distinguishable from those in control group. These suggest that OLP might be a predisposing condition to Candida infection and certain specific genotypes of $C$. albicans are probably involved in occurrence and progression of OLP.

Generally, the prevalence of $C$. albicans in OLP patients differs from that found in healthy individuals. It has been reported that a positive Candida culture is more prevalent among OLP patients (48.9\%) than among control subjects (26.7\%) [13]. In the present study, we also identified a definitely higher positive frequency of C. albicans in the NEOLP group and E-OLP group compared to healthy individuals. This discrepancy might cause the dysfunction of lymphocytes in OLP. As Simark-Mattsson et al showed the proliferation and cytokines production of peripheral blood mononuclear cells from OLP were significantly reduced following the stimulation of $C$. albicans, thus reflecting a potential immune regulatory mechanism of OLP modulating by $C$. albicans [9]. The high prevalence of specific C. albicans isolates in OLP suggest that they might potentially have strong adaptability to the microenvironment under the dynamic interaction between OLP progression and the fungal strains [21]. Yet, this is not consistent with results reported by Artico et al, who found that the positive prevalence of colonization by Candida spp. was higher in healthy subjects than in OLP [11]. The possible explanation for this 


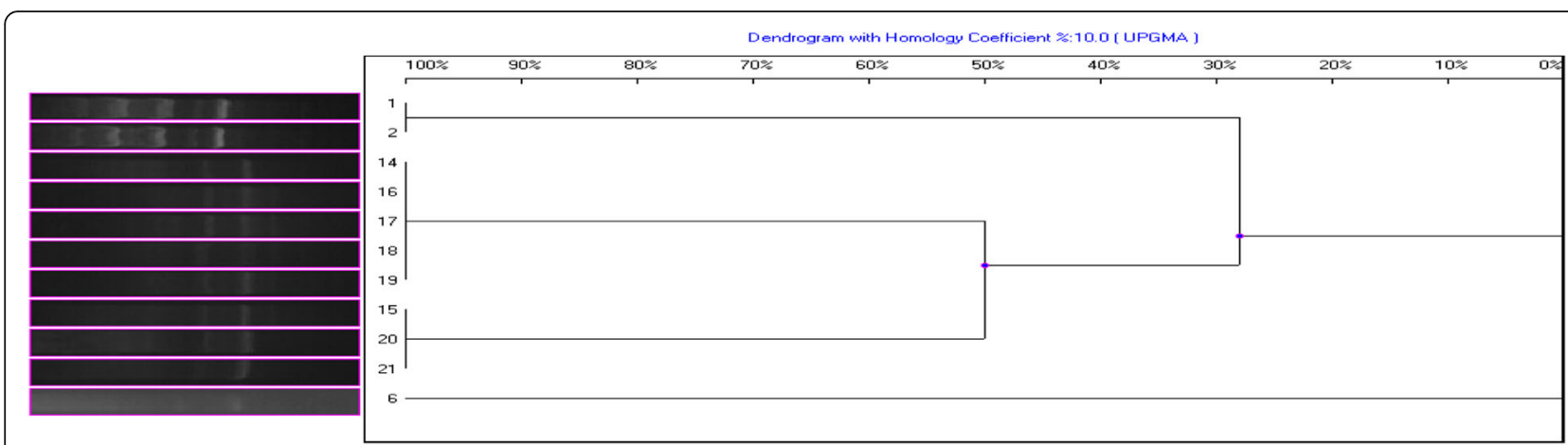

Fig. 3 Genotyping tree of gene homology coefficient $S_{A B}$ of $C$. albicans by UIV Band analysis. Horizontal axis $=S_{A B}$; Vertical axis $=$ genotypes of RAPD products of isolated strains. 1-2 =E-OLP strains no. 2, 8, $\mathrm{S}_{A B}=100 \% ; 14,16-19=$ NE-OLP strains no. 3-7 and 11, $\mathrm{S}_{A B}=100 \% ; 15,20,21=N E-$ OLP strains no. 9, 10, and 12, $S_{A B}=100 \% ; 6=$ No. 1 strain from the control group. (The numbers of strains did not relate to the numbers of cases).

discrepancy may lie in the differences of the sample size and methods including sample collection sites.

In the present study, the RAPD and ITS sequences were both chosen, because other studies failed to further classify C. albicans among different groups situation, with no better precision than telling just that they were C. albicans, and above reported two methods' results had a clear coherence and consistency. In fact, it is a well-known fact that phenotyping is vulnerable to being influenced by environment and involved in poor repeatability, weak identification, and instability [22]. In contrast, genotyping improves the precision of $C$. albicans species and subtypes [23]. What supports the importance of genotyping is that $C$. albicans does not differ in phenotype between dimorphologicly respective symbiotic and pathogenic situations.

Although other technologies as multilocus sequence typing and microsatellite typing are thought to be more efficient and reliable, and even if the RAPD and ITSPCR are viewed as obsolete by some scholars, we found them to be very useful. As a result, RAPD products showed by electrophoresis analysis that the healthy controls had only one band, E-OLP isolates had multiple bands, and all the eight NE-OLP isolates focused on two bands, and were further confirmed by ITS sequence. Moreover, the genetic homology coefficient $\mathrm{S}_{\mathrm{AB}}$ was less than 30\% between the control, NE-OLP, and E-OLP groups, while it was $100 \%$ inside subgroup. Previous genotyping results have usually revealed that $C$. albicans infection in OLP are exogenous [24], while authors here think that if they were exogenous, they should be disordered or similar in E-OLP or NE-OLP instead of the ordered genotypes. In addition, the exogenous colonization of C. albicans should reveal some indefiniteness and randomness. Nonetheless, a common natural symbionts C. albicans in OLP has not yet been found, which is why we are more inclined toward our initial hypothesis. On the contrary, we assumed that it was the endogenous genotypic changes of oral C. albicans under special environmental and ecological conditions that led to development of OLP. This might be because the specific microenvironment fostered a mutation of $C$. albicans from symbionts to further specific pathogenic genotypes which is also supported by other researches $[15,21,22]$

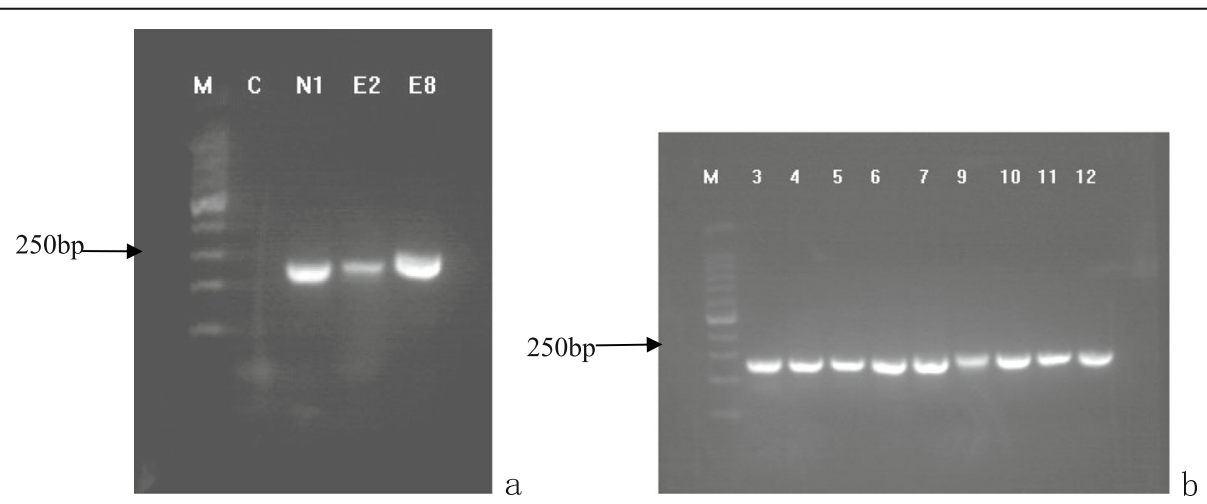

Fig. 4 Electrophoresis patterns of ITS PCR results (all $250 \mathrm{bp}$ ) of strains. 4a: N1 strain from the control group, and E2 and E8 strains from the E-OLP group. 4b: 3-7 and 9-12 strains from the NE-OLP group. (The numbers of strains did not relate to the numbers of cases) 


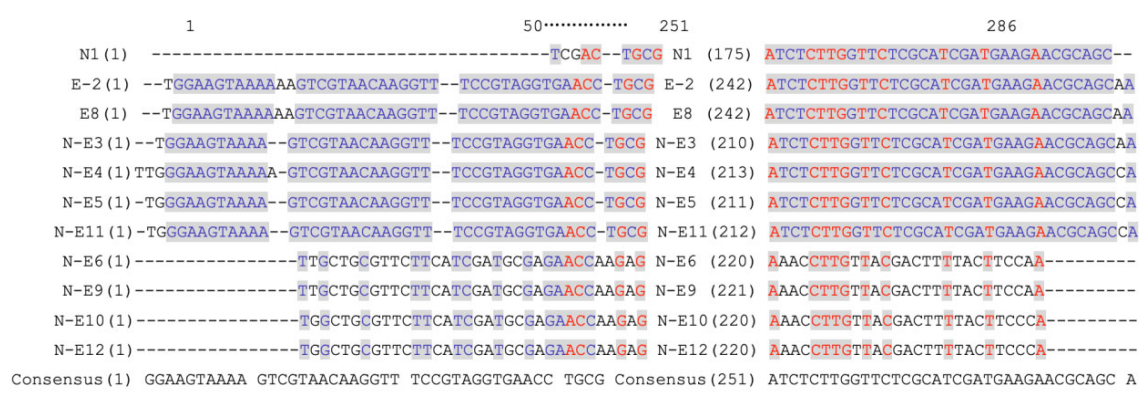

Fig. 5 Sequences analysis results using Vector NTI Suite. 5'-TCGACTGC...AAGAACGCAGC-3' was named as Type I for no. 1 strain from the control group of 101 subjects; 5'-GGAAGTAA...GAACGCAGCCA-3' was named as Type lla for strains no. 3-5, 7 and 11 from the NE-OLP group of 96 cases; 5'-TGGCTGCG...TTTACTTCCCA-3' was named as Type Illb for strains no. 6, 9, 10 and 12 from the NE-OLP group of 96 cases; 5'-TGGAAGTA.. GAACGCAGCAA-3' was named as Type III for strains no. 2 and 8 from the E-OLP group of 53 cases. (The numbers of strains did not relate to the numbers of cases)

and hopefully future molecular epidemiology, and to induce a T-cell-mediated immune response. As for elements of microenvironmental oral cavity including $\mathrm{PH}$, temperature and ingredients in salivary or gum fluids, they have been identified to induce pathogen's virulence key gene mutation and immune reaction or new balance [25]. Also, it is possible that the mutation drives C. albicans to get involve in and better adapt to a current environment [26], thus forming the type of dominant pathogen, which eventually affects the severity of the disease upon its interaction with the host. In this study, the results of both RAPD and ITS sequence showed that both intra-species homology and inter-species variations existed in C. albicans among the three clinical groups. The correlation between pathogen mutation and clinical progression of OLP was reflected in the changes from type I for normal strains, to type IIa, type IIb for NEOLP, and to type III for E-OLP strains. Besides the underlying implication that an endogenous mutation of Candida might be the key to uncovering the etiology of OLP, these findings can potentially be used as indicator for the severity evaluation for OLP, as well as a therapeutic basis for individualized treatment.

Although previous studies have reported that OLP attracts C. albicans' collection in lesions [15], we assume that Candida is probably the initial pathogen and the antigen for OLP (Fig. 6), since that OLP can be easily cured by addressing oral hygiene and dental health problems, which quite often involve fungi. If not so, solving the oral hygiene, dental and oral health, consuming antifungal drugs should have never worked. From these clinical aspects, the curative effect is comparatively easier in treating E-OLP than NE-OLP with superficial consumption of corticosteroids, and then subsequently or simultaneously addressing dental and hygiene problems same as treating NE-OLP. Correspondingly, overlooked patients' poor oral hygiene and health habits are indeed the cause for substantially prolonged healing and recurrences of OLP showing in our daily work and also mentioned in other studies. In fact, practice experience tells us that addressing oral hygiene and dental problems (which tend to involve numerous fungi and Candida) is a most effective way to cure OLP in clinical work with more accuracy than other therapies such as adoption of immune drugs or herb products and similar medicine.

Accordingly, a strong positive relationship has also been identified between the Candida infection in oral cavity and the degree of epithelial dysplasia or OSCC [3, 27]. Gainza-Cirauqui and colleagues suggested that $C$. albicans isolated from potentially carcinogenic oral diseases could produce mutagenic amounts of acetaldehyde, which are involved in abnormal epithelial proliferation of mucosa [28]. This further induced adverse events like disorders and formation of strange cellular keratin that deteriorates the abnormal nuclear divisions and cell proliferation in epithelial plaque, thus developing to cancers [29]. Therefore, clinicians should pay special attention to the presence of Candida altering from combionts to pathogenic, and any premalignant dental-to-mucosa friction injury when treating patients with OLP and OLP with epithelial dysplasia or carcinoma.

Our results with the clinical C. albicans strains confirmed that the ITS sequences and RAPD homology coefficient $\mathrm{S}_{\mathrm{AB}}$ of $C$. albicans were obviously different among E-OLP, NE-OLP and healthy individuals, thus suggesting that endogenous $C$. albicans in oral environment may be involved in the etiology and pathogenesis of OLP. Although the sample of strains in this study was small, and the mutual effect between OLP lesion and C. albicans gene mutation is dynamic and complex, reported results are strong. Nonetheless, future studies with larger sample size or an OLP animal model are required to further verify reported findings. 

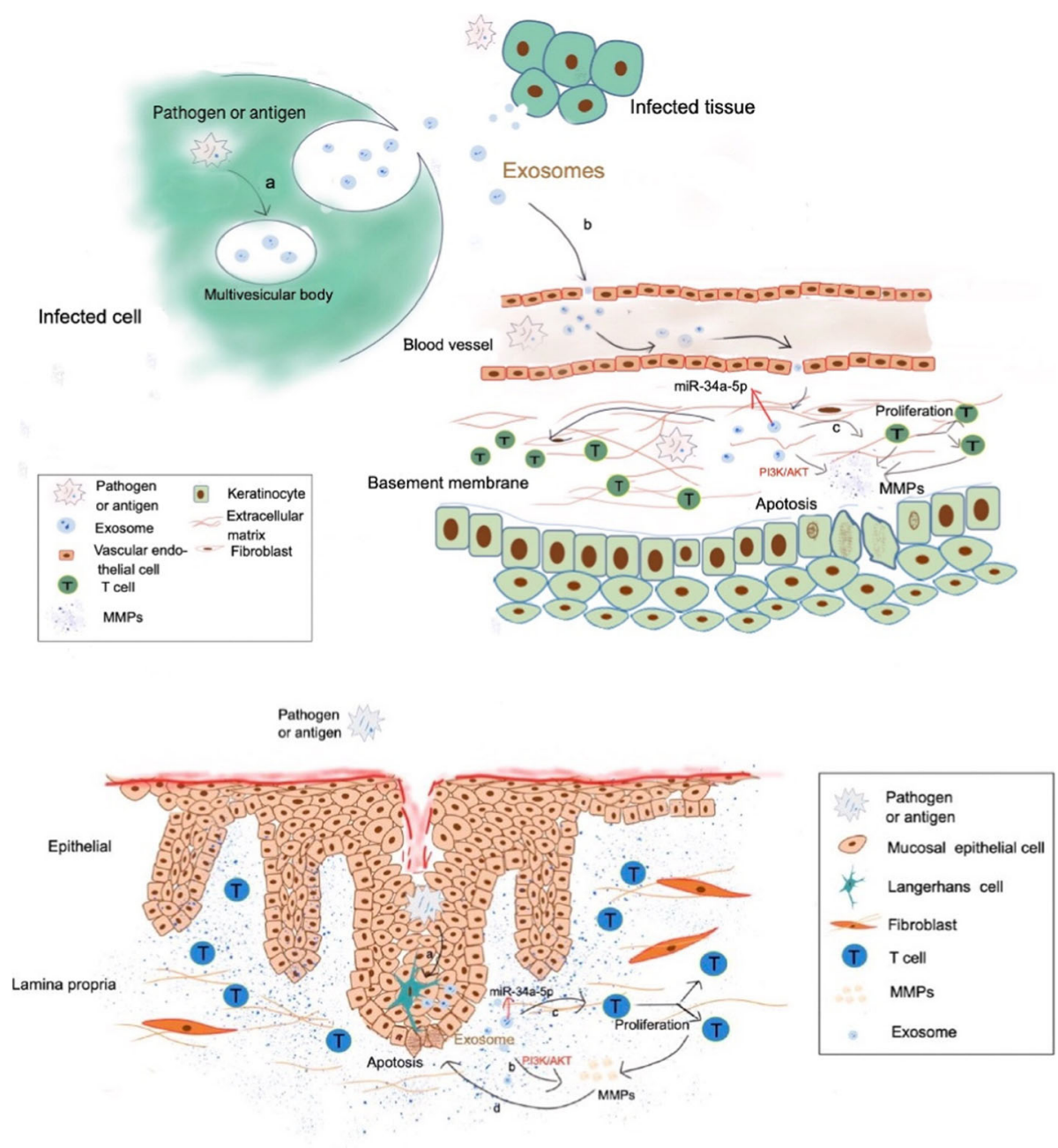

Fig. 6 This figure further illustrates the possible pathogen etiology for OLP. The authors view fungi e.g. Candida pathogen/antigen as a vital part of the etiology and pathogenesis for OLP. This figure is the authors own as Hong He et al. illustrated in p 5316-5317 of 5313-5323 American Journal of Translational Research, 2019,11(9), about pathogen/antigen acts via a, b, c molecular affairs in possible pathogenesis of OLP. Also please kindly acknowledge there is also a small "a" among epithelia cells

\section{Conclusions}

The etiology of OLP might be explained by the endogenous infection of Candida and its gene mutation under specific and dynamic microenvironment of the patient's oral cavity. These results should be further used to confirm the real etiology, as well as more precise and effective therapeutic strategies for OLP. This is crucial for correct intervention of premalignant progression in oral health.

\section{Abbreviations}

API: Analytical Profile Index; BLAST: Basic Local Alignment Search Tool; C. albicans: Candida albicans; DNA: DeoxyriboNucleic Acid; E-: Erosive-; IRB: Institutional review boards; ITS: Internal transcribed spacer; NE-: Nonerosive-: OLP: Oral lichen planus; OSCC: Oral squamous cell carcinoma: PCR: Polymerase chain reaction; RAPD: Random amplified polymorphic DNA

\section{Acknowledgements}

We are very grateful to the software specialist Professor Jun Liang from the IT department 2nd affiliated hospital medical school Zhejiang University for statistic assistance and additional check, and MedSci for English-language revision. We acknowledge all funding sources. All of the authors wish to thank Mengyun Zhang's help with literature collection and reading, and data statistic administration.

\section{Authors' contributions}

$H H, Y H P, Z M Y$ and $X X Y$ drafted the manuscript. $H H$ made critical revisions to include important intellectual content in the manuscript. YHP and PQ conducted the experiments, formatted and prepared the manuscript. All authors read and approved the final version.

\section{Funding}

The work was supported by the Teaching Reform Project of Zhejiang University School of Medicine yxyb20172030, National and provincial Health Commission Co-construction Fund WKJ-ZJ-1623, National Health Commission Public Welfare Fund 201502018, National Key R\&D Program of China 
2016YFC0902702, fund 2008C33026 [2011]666 from Science and technology Office of Zhejiang Province, 2016 fund for 151 talents second level Zhejiang Province 2014-2017, Scientific Research Foundation for the Returned Oversea Chinese Scholar J20120036 from HR Office of Zhejiang Province, Baoshi Oversea Scholarship zups1507 2013-2015, China Scholarship Council [2009]3004, and Research Program 2013KYB247 from Zhejiang Hygiene Bureau.

\section{Availability of data and materials}

The authors would deliver their raw data to him who any reader contacts asking for raw data.

\section{Ethics approval and consent to participate}

All procedures performed in studies involving human participants were in accordance with the ethical standards of the institutional and national research committee and with the 1964 Helsinki declaration and its later amendments or comparable ethical standards. Informed consents were obtained from all individual participants included in the study. Under the ethics committee of the Affiliated Second Hospital, and the ethics committee of the Affiliated Stomatology Hospital, Zhejiang University School of Medicine, China, all patients provided written informed consent. The protocols were reviewed and approved by the local institutional review boards (IRB). The IRB numbers for our medical ethic files were No. 2015 (17) of the stomatology hospital and 2010 (137) of the second hospital both affiliated to School of Medicine Zhejiang University.

\section{Consent for publication}

Consent for publication was obtained from all the patients.

\section{Competing interests}

The authors declare that they have no conflict of interest in this work.

\section{Author details}

'The Affiliated Stomatology Hospital, Zhejiang University School of Medicine, Yan'an Road, Hangzhou 310006, China. ${ }^{2}$ Key Laboratory of Oral Biomedical Research of Zhejiang Province, Hangzhou, China. ${ }^{3}$ School of Medicine, Zhejiang University, Hangzhou, China.

Received: 12 September 2019 Accepted: 10 February 2020

Published online: 14 March 2020

\section{References}

1. Ruokonen HMA, Juurikivi A, Kauppila T, Heikkinen AM, SeppanenKaijansinkko R. High percentage of oral lichen planus and lichenoid lesion in oral squamous cell carcinomas. Acta Odontol Scand. 2017;75:442-5.

2. Atzori L, Robustelli Test E, Ferreli C, Pilloni L, Rongioletti F. Impressive oral lichen planus progression to invasive squamous cell carcinoma in a three months lapse. Oral Oncol. 2019:90:150-2.

3. Mohd Bakri M, Mohd Hussaini H, Rachel Holmes A, David Cannon R, Mary RA. Revisiting the association between candidal infection and carcinoma, particularly oral squamous cell carcinoma. J Oral Microbiol. 2010;2. https:// doi.org/10.3402/jom.v2i0.5780.

4. Peng Q, Zhang J, Ye X, Zhou G. Tumor-like microenvironment in oral lichen planus: evidence of malignant transformation? Expert Rev Clin Immunol. 2017:13:635-43.

5. Roopashree MR, Gondhalekar RV, Shashikanth MC, George J, Thippeswamy $\mathrm{SH}$, Shukla A. Pathogenesis of oral lichen planus--a review. J Oral Pathol Med. 2010;39:729-34.

6. Kurago ZB. Etiology and pathogenesis of oral lichen planus: an overview. Oral Surg Oral Med Oral Pathol Oral Radiol. 2016;122:72-80.

7. Pippi R, Romeo U, Santoro M, Del Vecchio A, Scully C, Petti S. Psychological disorders and oral lichen planus: matched case-control study and literature review. Oral Dis. 2016;22:226-34.

8. Sudbery PE. Growth of Candida albicans hyphae. Nat Rev Microbiol. 2011;9: 737-48.

9. Simark-Mattsson C, Eklund C. Reduced immune responses to purified protein derivative and Candida albicans in oral lichen planus. J Oral Pathol Med. 2013:42:691-7.

10. Masaki M, Sato T, Sugawara Y, Sasano T, Takahashi N. Detection and identification of non-Candida albicans species in human oral lichen planus. Microbiol Immunol. 2011;55:66-70.
11. Artico G, Freitas RS, Santos Filho AM, Benard G, Romiti R, Migliari DA. Prevalence of Candida spp., xerostomia, and hyposalivation in oral lichen planus--a controlled study. Oral Dis. 2014;20:e36-41.

12. Arora S, Verma M, Gupta SR, Urs AB, Dhakad MS, Kaur R. Phenotypic variability and therapeutic implications of Candida species in patients with oral lichen planus. Biotech Histochem. 2016;91:237-41.

13. Bokor-Bratic M, Cankovic M, Dragnic N. Unstimulated whole salivary flow rate and anxiolytics intake are independently associated with oral Candida infection in patients with oral lichen planus. Eur J Oral Sci. 2013;121:427-33.

14. Xiang Y, Zhu X, Huang Q, Zheng J, Fu W. Real-time monitoring of mycobacterium genomic DNA with target-primed rolling circle amplification by a au nanoparticle-embedded SPR biosensor. Biosens Bioelectron. 2015;66:512-9.

15. Zeng X, Xiong C, Wang Z, Jiang L, Hou X, Shen J, et al. Genotypic profiles and virulence attributes of Candida albicans isolates from patients with oral lichen planus. APMIS. 2008;116:284-91.

16. Koffi YF, Diguta C, Alloue-Boraud M, et al. PCR-ITS-RFLP identification of pineapple spoilage fungi[J]. Rom Biotechnol Lett. 2019;24:418-24.

17. Chow S, Suzuki S, Matsunaga T, et al. Investigation on natural diets of larval marine animals using peptide nucleic acid-directed polymerase chain reaction clamping[J]. Mar Biotechnol. 2011;13:305-13.

18. Dassanayake RS, Ellepola AN, Samaranayake YH. LP S Phenotypic diversity of oral $C$ albicans isolated on single and sequential visits in an HIV-infected Chinese cohort. APMIS. 2002;110:329-37.

19. Huys G, Vancanneyt M, D'Haene K, Vankerckhoven V, Goossens H, Swings J. Accuracy of species identity of commercial bacterial cultures intended for probiotic or nutritional use. Res Microbiol. 2006;157:803-10.

20. Stender H, Fiandaca M, Hyldig-Nielsen JJ. J C. PNA for rapid microbiology. J Microbiol Methods. 2002:48:1-17.

21. Zeng $X$, Hou $X$, Wang $Z$, Jiang $L$, Xiong $C$, Zhou $M$, et al. Carriage rate and virulence attributes of oral Candida albicans isolates from patients with oral lichen planus: a study in an ethnic Chinese cohort. Mycoses. 2009:52:161-5.

22. Macura AB, Voss A, Melchers WJG, Meis JFGM, Syslo J, Heczko PB. Characterization of Pathogenetic determinants of Candida albicans strains. Zentralblatt für Bakteriologie. 1998;287:501-8.

23. Luu LN, Cowen LE, Sirjusingh C, Kohn LM, Anderson JB. Multilocus genotyping indicates that the ability to invade the bloodstream is widespread among Candida albicans isolates. J Clin Microbiol. 2001;39:1657-60.

24. De Carli JP, da Silva SO, De Carli BMG, Zanata A, Trentin MS, Linden MSS, et al. Clinical Correlation of Oral Candidosis and Oral Lichen Planus. Oral Candidosis; 2015. p. 75-82

25. He H, Cong Y, Yang H, Dong Y. Mutative expression in Candida albicans infection and cytokine signaling network in gene knockout mice. Eur J Clin Microbiol Infect Dis. 2010;29:913-6.

26. Wang JM, Bennett RJ, Anderson MZ. The genome of the human pathogen Candida albicans is shaped by mutation and cryptic sexual recombination. MBio. 2018;9:e01205-18.

27. McCullough M, Jaber M, Barrett AW, Bain L, Speight PM, SR P. Oral yeast carriage correlates with presence of oral epithelial dysplasia. Oral Oncol. 2002;38:391-3.

28. Gainza-Cirauqui ML, Nieminen MT, Novak Frazer L, Aguirre-Urizar JM, Moragues MD, Rautemaa R. Production of carcinogenic acetaldehyde by Candida albicans from patients with potentially malignant oral mucosal disorders. J Oral Pathol Med. 2013;42:243-9.

29. Moyes DL, Shen C, Murciano C, Runglall M, Richardson JP, Arno M, et al. Protection against epithelial damage during Candida albicans infection is mediated by PI3K VAkt and mammalian target of rapamycin signaling. J Infect Dis. 2014;209:1816-26.

\section{Publisher's Note}

Springer Nature remains neutral with regard to jurisdictional claims in published maps and institutional affiliations. 